\title{
Adverse Pregnancy Outcomes Following Motor Vehicle Crashes
}

\author{
Catherine J. Vladutiu, PhD, Stephen W. Marshall, PhD, Charles Poole, ScD, Carri Casteel, \\ PhD, M. Kathryn Menard, MD, and Harold B. Weiss, PhD \\ Department of Epidemiology (Vladutiu, Marshall, Poole, Casteel), Gillings School of Global Public \\ Health, the Injury Prevention Research Center (Vladutiu, Marshall, Casteel), the Department of \\ Obstetrics \& Gynecology (Menard), School of Medicine, University of North Carolina, Chapel Hill, \\ North Carolina, United States; the Injury Prevention Research Unit (Weiss), Department of \\ Preventive and Social Medicine, Dunedin School of Medicine, University of Otago, New Zealand
}

\begin{abstract}
Background-Motor vehicle crashes are a leading cause of serious trauma during pregnancy, but little is known about their relationships with pregnancy outcomes.
\end{abstract}

Purpose-To estimate the association between motor vehicle crashes and adverse pregnancy outcomes.

\begin{abstract}
Methods-A retrospective cohort study of 878,546 pregnant women, aged 16-46 years, who delivered a singleton infant in North Carolina (NC) from 2001 to 2008. Pregnant drivers in crashes were identified by probabilistic linkage of vital records and crash reports. Poisson regression modeled the association among crashes, vehicle safety features, and adverse pregnancy outcomes. Analyses were conducted in 2012.
\end{abstract}

Results-In 2001-2008, 2.9\% of pregnant NC women were drivers in one or more crashes. After a single crash, compared to not being in a crash, pregnant drivers had slightly elevated rates of preterm birth (adjusted rate ratio, aRR $=1.23,95 \% \mathrm{CI}=1.19,1.28$ ); placental abruption ( $\mathrm{aRR}=1.34$, $95 \% \mathrm{CI}=1.15,1.56$ ); and premature rupture of the membranes (PROM; $\mathrm{aRR}=1.32,95 \% \mathrm{CI}=1.21$, 1.43). Following a second or subsequent crash, pregnant drivers had more highly elevated rates of preterm birth $(\mathrm{aRR}=1.54,95 \% \mathrm{CI}=1.24,1.90)$; stillbirth $(\mathrm{aRR}=4.82,95 \% \mathrm{CI}=2.85,8.14)$; placental abruption $(\mathrm{aRR}=2.97,95 \% \mathrm{CI}=1.60,5.53)$; and PROM ( $\mathrm{aRR}=1.95,95 \% \mathrm{CI}=1.27,2.99)$. Stillbirth rates were elevated following crashes involving unbelted pregnant drivers $(\mathrm{aRR}=2.77$, $95 \% \mathrm{CI}=1.22,6.28)$ compared to belted pregnant drivers.

Conclusions-Crashes while driving during pregnancy were associated with elevated rates of adverse pregnancy outcomes, and multiple crashes were associated with even higher rates of adverse pregnancy outcomes. Crashes were especially harmful if drivers were unbelted.

\section{Introduction}

Trauma during pregnancy is a leading cause of maternal and fetal morbidity and mortality. In the U.S., it has been estimated that up to $7 \%$ of all pregnancies are complicated by

\footnotetext{
(C) 2013 American Journal of Preventive Medicine. Published by Elsevier Inc. All rights reserved. Address correspondence to: Catherine J. Vladutiu, PhD, Department of Epidemiology, Gillings School of Global Public Health, University of North Carolina at Chapel Hill, 137 E. Franklin Street, CB \#8050, Chapel Hill NC 27599-8050. cvladutiu@ unc.edu. Publisher's Disclaimer: This is a PDF file of an unedited manuscript that has been accepted for publication. As a service to our customers we are providing this early version of the manuscript. The manuscript will undergo copyediting, typesetting, and review of the resulting proof before it is published in its final citable form. Please note that during the production process errors may be discovered which could affect the content, and all legal disclaimers that apply to the journal pertain.

No other financial disclosures have been reported by the authors of this paper.
} 
traumatic injury. ${ }^{1}$ Blunt abdominal trauma is of particular concern to a pregnant woman and her fetus since it can directly and indirectly harm fetal organs as well as shared maternal and fetal organ systems. Direct fetal injury can include splenic rupture, skull fractures and brain injury; direct harm to shared organs and systems includes placental abruption, uterine rupture, and amniotic rupture. ${ }^{2-4}$ Fetuses may also be vulnerable to indirect effects of trauma, such as an increased risk of spontaneous preterm birth or low birth weight resulting from premature labor, with consequences that can have long-term effects. ${ }^{4,5}$

Motor vehicle crashes are responsible for most hospitalized trauma during pregnancy, but little is known about their impact on fetal morbidity and mortality. ${ }^{4-9}$ While several case reports have quantified the effect of crashes on individual fetal outcomes, ${ }^{10-12}$ populationbased studies are few, largely due to the lack of standardized reporting of pregnancyassociated crashes and crash-related fetal outcomes. State motor vehicle crash reports do not routinely report pregnancy status or crash-related fetal deaths and pregnancy records often lack information on crash history.

To overcome these limitations, researchers have used record linkage methods to match vital records and crash reports in order to examine the association between police-reported crashes and adverse fetal outcomes. Only three linkage studies have been conducted and all have had relatively small study populations. ${ }^{13-15}$ In addition, only one study compared fetal outcomes for pregnant women in crashes to those not in crashes, ${ }^{13}$ and no studies have examined the dose-response effects of multiple crashes on pregnancy outcomes. Populationbased studies with larger sample sizes are needed to more precisely estimate the effect of multiple crashes and vehicle safety features on pregnancy outcomes. The objective of this study was to use a large cohort of pregnant women from North Carolina (NC) to estimate the association among crashes, seat belt use, airbag availability, and selected adverse fetal outcomes and obstetric conditions.

\section{Methods}

\section{Study population}

This retrospective cohort study examined 878,546 pregnant NC residents, aged 16-46 years, who reached the 20th week of pregnancy and delivered a live or stillborn singleton infant between January 1, 2001 and December 31, 2008. These women completed a total of $115,797,259$ pregnancy days following the 20th week of pregnancy. They were identified from live birth and fetal death records $(n=993,274)$. Pregnancies that did not reach the 20th week were excluded because fetal deaths occurring before 20 weeks are not reported in NC vital records. Records were excluded for women aged <16 years $(n=7075)$ since driver crashes in this pre-licensure age group are rare and for women older than 46 years at delivery $(n=237)$ and those with multiple gestation deliveries $(n=33,360)$ since older maternal age and multi-fetal gestation are associated with a higher risk of adverse pregnancy outcomes. Records with missing data for at least one of the following: mother's age, multiple gestation status, or gestational age at delivery, were removed $(n=603)$. Records were also excluded for 73,453 pregnant women who did not meet at least one of the cohort inclusion criteria, including those who completed 20 or more weeks of pregnancy before $1 / 1 / 2001(n=40,014)$; became pregnant less than 45 weeks before $1 / 1 / 2009(n=14,032)$; were aged $<16$ years after the 20th week of pregnancy was completed $(n=3093)$; delivered a liveborn infant before the 20th week of pregnancy $(n=455)$; or were nonresidents of NC $(n=17,151)$. These inclusion criteria are not mutually exclusive, thus some records are counted in multiple categories.

To determine if pregnant women were drivers in motor vehicle crashes, individual vital records were probabilistically linked to state crash records using mother's first, middle and 
last names, date of birth, race, and residential county. Passengers and unlicensed NC drivers in crashes could not be linked to vital records because the crash reports contained identifying information only for licensed drivers. Linked record pairs were ranked from highest to lowest based on their match weights and probabilities. The expected number of false positives was estimated for each pair by summing 1-match probability. A false positive rate of $1 \%$ was selected a priori and matched pairs were selected one-by-one until this desired rate was obtained. The median match probability was 0.9999 with an interquartile range of $9.88 \times 10^{-4}$. This linkage was performed in 2010-2011 using LinkSolv generalized linkage software (Strategic Matching Inc.).

\section{Measures}

Motor vehicle crashes-A motor vehicle crash was defined as a crash that involved a NC licensed female driver of a motor vehicle or passenger truck. Crash reports are completed by police officers if the crash occurred on a public roadway and resulted in at least one of the following: fatality or nonfatal personal injury to any vehicle occupant, total property damage greater than $\$ 1000$, or property damage of any amount to a vehicle seized. A woman could be a driver in more than one crash during the same pregnancy. Motor vehicle crashes were classified into three categories according to the number of crashes a woman experienced during each pregnancy (no crashes, 1st, and 2nd or subsequent crashes).

Vehicle safety features-Belt use was defined as shoulder and/or lap belt use as reported by the investigating officer at the crash scene. Police officers also reported whether or not a motor vehicle was equipped with an airbag at the time of the crash and whether or not it deployed. In this study, airbag availability was classified as equipped and not equipped, regardless of deployment status.

Pregnancy outcomes-Preterm birth was defined as a live birth that occurred between 20 and 37 weeks of gestation. To determine if a birth was preterm, gestational age was estimated using the National Center for Health Statistics' method for estimating gestational age in U.S. vital statistics. ${ }^{16,17}$ This methodology relies primarily on the self-reported date of the last menstrual period (LMP). For records that were missing the LMP date or had an implausible gestational age when compared to birth weight, the clinical estimate was used ( $n=51,593$ or $5.2 \%$ ). If records were missing the LMP-based and clinical estimates, the physician's estimate (estimated from pregnancy history, early ultrasound, or examination), which is reported only on fetal death records, was used for stillbirths $(n=531,<0.1 \%)$. Records missing all values were excluded $(n=481,<0.1 \%)$.

Stillbirth was defined as an intrauterine death that occurred after the 20th week of gestation. Stillbirth status, excluding induced abortions, was determined by hospital administrators, physicians, and medical examiners.

Obstetric complications, as recorded on the live birth and fetal death certificates, were placental abruption (i.e., separation of the placenta from the uterus during pregnancy) and premature rupture of the membranes (PROM) (i.e., spontaneous rupture of the amniochorionic membrane occurring 12 or more hours before the onset of labor).

\section{Covariates}

Potential covariates included maternal age, maternal race and Hispanic ethnicity (categorized as non-Hispanic white, non-Hispanic black, other non-Hispanic race, and Hispanic), maternal education, prenatal tobacco use, prenatal alcohol use, trimester of prenatal care initiation, and parity (defined as the total number of prior live births). Data for 
these covariates were obtained from vital records. Vehicle model year, as indicated in the crash reports, was selected for examination in the airbag analysis.

\section{Statistical analysis}

Poisson regression was used to estimate rate ratios for the association between crashes and preterm birth, stillbirth, placental abruption, and PROM. ${ }^{18}$ Incidence rates were estimated because they can take into account the variability in the timing of events. This method accounted for the time-dependent nature of an exposure (i.e., motor vehicle crashes) that changed over the course of pregnancy and allowed pregnancy outcomes to be modeled for pregnancies with varying lengths, including those with shorter duration and limited potential for crash exposure. Person-time at risk was defined as pregnancy days completed after the 20th week. Since the number of crashes was modeled as a time-dependent exposure, an individual woman could contribute time to more than one crash exposure category if she was a driver in more than one crash during the same pregnancy. Tests for trend were conducted by modeling the number of crashes as a continuous variable.

Rate ratios were also estimated for the association among seat belt use, airbag availability and pregnancy outcomes only among pregnant drivers in crashes. For pregnant drivers who were in at least one crash before the 20th week, person-time at risk was defined as pregnancy days completed after the 20th week. For those in crashes only after the 20th week, person-time at risk was defined as pregnancy days completed after the first crash. Vehicle safety features were modeled as time-dependent exposures, thus an individual woman could contribute time to more than one exposure category if she was in more than one crash.

Incidence rates were defined as the number of events (i.e., preterm birth, stillbirth, placental abruption, or PROM) divided by the total person-time at risk (counted in pregnancy days), within each exposure category. For preterm birth rates, only live births and pregnancy days occurring between 20 and 37 weeks were counted. Rates for all other outcomes included all events and days that occurred after the 20th week. Rate ratios for the crash analysis were adjusted for covariates identified a priori from the literature ${ }^{13,19}$ as being associated with crashes and adverse pregnancy outcomes, including maternal age, prenatal tobacco use, prenatal alcohol use, trimester of prenatal care initiation (modeled as a time-varying covariate), and parity. Rate ratios for the seatbelt analysis were adjusted for maternal age and prenatal care initiation; rate ratios for the airbag analysis were adjusted for maternal age, seat belt use, and vehicle model year. All analyses were conducted in 2012. This study was approved by the Institutional Review Board at the University of North Carolina at Chapel Hill.

\section{Results \\ Study population}

There were 25,168 pregnant $\mathrm{NC}$ women who were licensed drivers involved in one or more crashes during pregnancy in 2001-2008 (2.9\%); 24,399 women were drivers in only one crash $(2.8 \%)$, and 769 were in two or more crashes during the same pregnancy $(0.1 \%)$. A high proportion of pregnancy-time following the first crash was among women who were aged 18-34 years, non-Hispanic white, high school graduates, married, nontobacco users, nondrinkers, early initiators of prenatal care, and primiparas (Table 1). The distribution of pregnancy-time following the second or subsequent crashes was similar for most maternal characteristics, with the exception of age and marital status, where a high proportion of pregnancy-time was among women who were aged 18-24 years and unmarried. 


\section{Crashes and pregnancy outcomes}

Between 2001 and 2008, there were 100,515 preterm births (11.4\%) and 5,447 stillbirths $(0.6 \%)$ that occurred among pregnant women in the study population (Table 2). Pregnant drivers had elevated rates of preterm birth following their first crash, compared to no crashes. The highest rates were observed following their second or subsequent crashes. Each additional crash (i.e., an increase of one crash in the exposure measure) was associated with an increased rate of preterm birth (adjusted rate ratio $=1.23,95 \%$ CI, 1.19, 1.28). Similarly, pregnant drivers had higher rates of stillbirth following their first crash, and even higher rates following their second or subsequent crashes, compared to no crashes. The estimated rate ratio of stillbirth for each additional crash was 1.25 (95\% $\mathrm{CI}=1.07,1.46)$.

There were 5866 placental abruption events $(0.7 \%)$ and 19,721 PROM events $(2.2 \%)$ that occurred between 2001 and 2008 (Table 2). Pregnant drivers had higher rates of placental abruption and PROM following their first crash, compared to no crashes. The highest rates of placental abruption and PROM were observed following their second or subsequent crashes, compared to no crashes. For each additional crash, the estimated rate ratios for placental abruption and PROM were 1.39 (95\% $\mathrm{CI}=1.21,1.59)$ and $1.33(95 \% \mathrm{CI}=1.23$, $1.43)$, respectively.

\section{Vehicle safety features and pregnancy outcomes}

Among pregnant NC drivers who were involved in one or more crashes ( $\mathrm{n}=25,168), 2 \%$ were reportedly unbelted and $18 \%$ were driving in vehicles without airbags in at least one crash during pregnancy. Rates of adverse pregnancy outcomes, particularly stillbirth, were higher following crashes among unbelted pregnant drivers, compared to belted pregnant drivers (Table 3). Rates of preterm birth and placental abruption were higher following crashes involving vehicles without airbags compared to those equipped with airbags.

\section{Disscussion}

Motor vehicle crashes involving a pregnant driver were associated with elevated rates of adverse pregnancy outcomes, including preterm birth, stillbirth, placental abruption, and PROM. The rates of these outcomes increased as the number of crashes increased. Although the strongest associations were observed for stillbirth, less than $1 \%$ of pregnancies resulted in this outcome. Only one previous linkage study has examined the association between police-reported motor vehicle crashes (any versus none) and adverse pregnancy outcomes. ${ }^{13}$ This study found a weak, positive association between crashes and the risk of preterm birth (odds ratio, OR, 1.02, 95\% $\mathrm{CI}=0.94,1.11$ ) and no association with placental abruption $(\mathrm{OR}=1.00,95 \% \mathrm{CI}=0.81,1.24)$ among pregnant women in Utah (UT). ${ }^{13}$ Weak, positive associations were also observed for other outcomes, including low birth weight $(\mathrm{OR}=1.03$, $95 \% \mathrm{CI}=0.94,1.14)$ and fetal distress $(\mathrm{OR}=1.09,95 \% \mathrm{CI}=0.98,1.21) .{ }^{13}$ The UT researchers did not report the association between crashes and the risk of stillbirth or PROM. The stronger associations observed in this study may be due to a larger sample size that was almost three times as large as the UT study ${ }^{13}$ or the higher rate of adverse pregnancy outcomes among pregnant women in $\mathrm{NC}$ as compared to UT. ${ }^{20,21}$

Non-seat belt use and the lack of airbags were associated with elevated rates of selected adverse pregnancy outcomes. Most notably, the stillbirth rate following a crash involving an unbelted pregnant driver was almost three times as high as the stillbirth rate following a crash involving a belted pregnant driver. There are only two linkage studies that have examined the effect of crash-related belt use on selected adverse pregnancy outcomes. ${ }^{13,14}$ In Washington State, unbelted pregnant drivers in crashes were at higher risk of delivering a stillborn infant (relative risk, $\mathrm{RR}=4.1,95 \% \mathrm{CI}=0.8,20.3$ ) and at lower risk of placental 
abruption $(\mathrm{RR}=0.9,95 \%=\mathrm{CI} 0.4,2.2)$, compared to belted pregnant drivers. ${ }^{14}$ The association between belt use and the risk of preterm birth or PROM was not reported. In contrast to our findings, in UT, nonbelt use (versus belt use) was not associated with preterm birth $(\mathrm{OR}=1.00,95 \% \mathrm{CI}=0.78,1.29)$ and negatively associated with placental abruption $(\mathrm{OR}=0.88,95 \%=\mathrm{CI} 0.44,1.76) .{ }^{13}$ The association between belt use and PROM was not reported and fetal death estimates were limited by small numbers of events. Only one previous linkage study has examined crash-related airbag availability and adverse pregnancy outcomes. ${ }^{15}$ This study, which included both drivers and front seat passengers, found a weak positive association between airbag availability and preterm birth $(\mathrm{RR}=1.1,95 \% \mathrm{CI}=0.8$,

1.6) and no association with placental abruption $(\mathrm{RR}=1.0,95 \% \mathrm{CI}=0.4,2.4)$. The association between airbag availability and the risk of PROM was not reported and fetal death estimates were limited by small numbers.

Overall, this study expands on these few previous studies by examining multiple crashes and their vehicle safety characteristics while providing estimated rates for several pregnancy outcomes in a larger cohort. To date, this is the largest state-based study that has examined the effects of crashes during pregnancy in a cohort of pregnant women. The largest previous study, conducted in UT, included 325,349 births (8,983 exposed to crashes). ${ }^{13}$ This is also the first study to examine dose-response effects of increasing number of crashes on the rate of adverse pregnancy outcomes. Record linkage allowed the ascertainment of both hospitalized and nonhospitalized pregnant driver crashes, thus allowing a population-based approach to examining the effect of crashes on fetal outcomes.

This study has several limitations. There is the potential for misclassification of pregnancy outcomes as determined from vital records data. LMP-based estimates of gestational age are often misclassified due to irregular menstrual cycles or errors in recall or data recording. ${ }^{17,22-24}$ This limitation was addressed by replacing implausible and missing LMPbased measures with clinical estimates, but misclassified estimates likely remain. The validity of reported obstetric conditions may be problematic, ${ }^{25,26}$ particularly in the presence of adverse birth outcomes. Without medical records, these outcomes could not be validated. Behavioral risk factors, including prenatal tobacco and alcohol use, may also be unreliable due to underreporting. ${ }^{25,26}$ Vital records do not include measures of socioeconomic status or other social and behavioral factors that may confound several of the associations that were observed. In the crash data, self-reported seat belt use is likely over-reported, especially since NC has a primary enforcement seat belt law. Thus, nondifferential misclassification may bias the estimated rates and underestimate the true association with adverse pregnancy outcomes. There may also be unmeasured risk factors associated with being in multiple crashes and pregnancy outcomes. Residual confounding by these factors and other unmeasured or poorly measured maternal and crash characteristics may have weakened the associations that were observed.

Due to the lack of information regarding early fetal losses and terminations in vital records, similar to the other linkage studies, all crashes and fetal outcomes occurring before the 20th week of pregnancy were not observed. Additionally, out-of-state crashes among pregnant $\mathrm{NC}$ drivers, unreported NC crashes (e.g., those occurring on private roads, without property damage or occupant injuries), and crashes involving unlicensed drivers or pregnant passengers in NC were not captured. Previous studies have found that crashes, particularly minor ones, are often underreported which may cause bias when examining crash-related outcomes. ${ }^{27,28}$ In this study, estimates could be biased if underreporting was differential by an unknown covariate that was also associated with adverse pregnancy outcomes. A sensitivity analysis was conducted and it was found that differential misclassification created negligible bias for selected associations (Appendix A, available online at www.ajpmonline.org). 


\section{Conclusion}

This study highlights the importance of crashes during pregnancy and their possible adverse effects on pregnancy outcomes. Clinicians should be aware of these effects and should advise pregnant women about the risk of being in a crash and the long-term consequences that crashes can have on their pregnancies. Given the associations that were observed, a better understanding of the circumstances surrounding crashes during pregnancy is needed to develop effective strategies for prevention.

\section{Supplementary Material}

Refer to Web version on PubMed Central for supplementary material.

\section{Acknowledgments}

The authors would like to thank Larry Cook at the University of Utah for his assistance with the probabilistic record linkage, Eric Rodgman and Carol Martell at the University of North Carolina Highway Safety Research Center for their help with crash data acquisition and preparation, and Matt Avery at the North Carolina State Center for Health Statistics for his help with vital records data acquisition and preparation.

CJV received partial support from grant T32-HD052468-03 from the Eunice Kennedy Shriver National Institute of Child Health and Human Development, National Institutes of Health, Bethesda, Maryland.

\section{References}

1. Peckham CH, King RW. A study of intercurrent conditions observed during pregnancy. Am J Obstet Gynecol. 1963; 87:609-624. [PubMed: 14074027]

2. Brown H. Trauma in pregnancy. Obstet Gynecol. 2009; 114(1):147-160. [PubMed: 19546773]

3. Pearlman MD, Tintinalli JE, Lorenz RP. Blunt trauma during pregnancy. N Eng J Med. 1990; 323(23):1609-1613.

4. El Kady D. Perinatal outcomes of traumatic injuries during pregnancy. Clin Obstet Gynecol. 2007; 50(3):582-591. [PubMed: 17762411]

5. El Kady D, Gilbert W, Anderson J, Danielsen B, Towner D, Smith L. Trauma during pregnancy: an analysis of maternal and fetal outcomes in a large population. Am J Obstet Gynecol. 2004; 190:1661-1668. [PubMed: 15284764]

6. Weiss HB, Sauber-Schatz EK, Cook LJ. The epidemiology of pregnancy-associated emergency department injury visits and their impact on birth outcomes. Accid Anal Prev. 2008; 40(3):10881095. [PubMed: 18460377]

7. Kuo C, Jamieson D, McPheeters M, Meikle S, Posner S. Injury hospitalizations of pregnant women in the United States, 2002. Am J Obstet Gynecol. 2007; 161:e1-e6. [PubMed: 17306664]

8. Schiff MA, Holt VL, Daling JR. Maternal and infant outcomes after injury during pregnancy in Washington State from 1989 to 1997. J Trauma. 2002; 53(5):939-945. [PubMed: 12435947]

9. Nannini A, Lazar J, Berg C, et al. Injury: a major cause of pregnancy-associated morbidity in Massachusetts. J Midwifery Women's Health. 2008; 53(1):3-10. [PubMed: 18164428]

10. Nguyen CS, Chase DM, Wing DA. Severe fetal skull fracture and death subsequent to a motor vehicle crash with frontal airbag deployment. J Trauma Inj Infect Crit Care. 2009; 67(6):e220 e221.

11. Weir LF, Pierce BT, Vazquez JO. Complete fetal transection after a motor vehicle collision. Obstet Gynecol. 2008; 111(2 Pt 2):530-532. [PubMed: 18239011]

12. Kiryabwire J, Chaseling R, Lang EW. Extensive in utero traumatic subarachnoid haemorrhage and abruptio placentae. J Trauma. 2005; 59(1):236-238. [PubMed: 16096570]

13. Hyde L, Cook L, Olson L, Weiss H, Dean J. Effect of motor vehicle crashes on adverse fetal outcomes. Obstet Gynecol. 2003; 102(2):279-286. [PubMed: 12907100] 
14. Wolf M, Alexander B, Rivara F, Hickok D, Maier R, Starzyk P. A retrospective cohort study of seatbelt use and pregnancy outcome after a motor vehicle crash. J Trauma. 1993; 34(1):116-119. [PubMed: 8437176]

15. Schiff MA, Mack CD, Kaufman RP, Holt VL, Grossman DC. The effect of air bags on pregnancy outcomes in Washington State: 2002-2005. Obstet Gynecol. 2010; 115(1):85-92. [PubMed: 20027039]

16. Wier ML, Pearl M, Kharrazi M. Gestational age estimation on United States live birth certificates: a historical overview. Paediatr Perinat Epidemiol. 2007; 21(Suppl 2):4-12. [PubMed: 17803613]

17. Martin JA. United States vital statistics and the measurement of gestational age. Paediatr Perinat Epidemiol. 2007; 21(Suppl 2):13-21. [PubMed: 17803614]

18. Frome EL, Checkoway H. Use of Poisson regression models in estimating incidence rates and ratios. Am J Epidemiol. 1985; 121(2):309-323. [PubMed: 3839345]

19. Schiff M, Holt V. Pregnancy outcomes following hospitalization for motor vehicle crashes in Washington State from 1989 to 2001. Am J Epidemiol. 2005; 161:503-510. [PubMed: 15746466]

20. National Center for Health Statistics. Final mortality data, 1990-1994, and period linked birth/ infant death data, 1995-present. 2009 www.marchofdimes.com/peristats.

21. National Center for Health Statistics. Final natality data. 2009 www.marchofdimes.com/peristats.

22. Ananth CV. Menstrual versus clinical estimate of gestational age dating in the United States: temporal trends and variability in indices of perinatal outcomes. Paediatr Perinat Epidemiol. 2007; 21(Suppl 2):22-30. [PubMed: 17803615]

23. David RJ. The quality and completeness of birthweight and gestational age data in computerized birth files. Am J Public Health. 1980; 70(9):964-973. [PubMed: 7406096]

24. Dietz PM, England LJ, Callaghan WM, Pearl M, Wier ML, Kharrazi M. A comparison of LMPbased and ultrasound-based estimates of gestational age using linked California live birth and prenatal screening records. Paediatr Perinat Epidemiol. 2007; 21(Suppl 2):62-71. [PubMed: 17803619]

25. Buescher P, Taylor K, Davis M, Bowling JM. The quality of the new birth certificate data: a validation study in North Carolina. Am J Public Health. 1993; 83(8):1163-1165. [PubMed: 8342728]

26. Vinikoor LC, Messer LC, Laraia BA, Kaufman JS. Reliability of variables on the North Carolina birth certificate: a comparison with directly queried values from a cohort study. Paediatr Perinat Epidemiol. 2010; 24(1):102-112. [PubMed: 20078836]

27. Elvik R, Mysen AB. Incomplete accident reporting: meta-analysis of studies made in 13 countries. Transportation Research Record. 1999; 1665:133-140.

28. Hauer E, Hakkert AS. Extent and some implications of incomplete accident reporting. Transportation Research Board. 1988; 1185:1-10. 


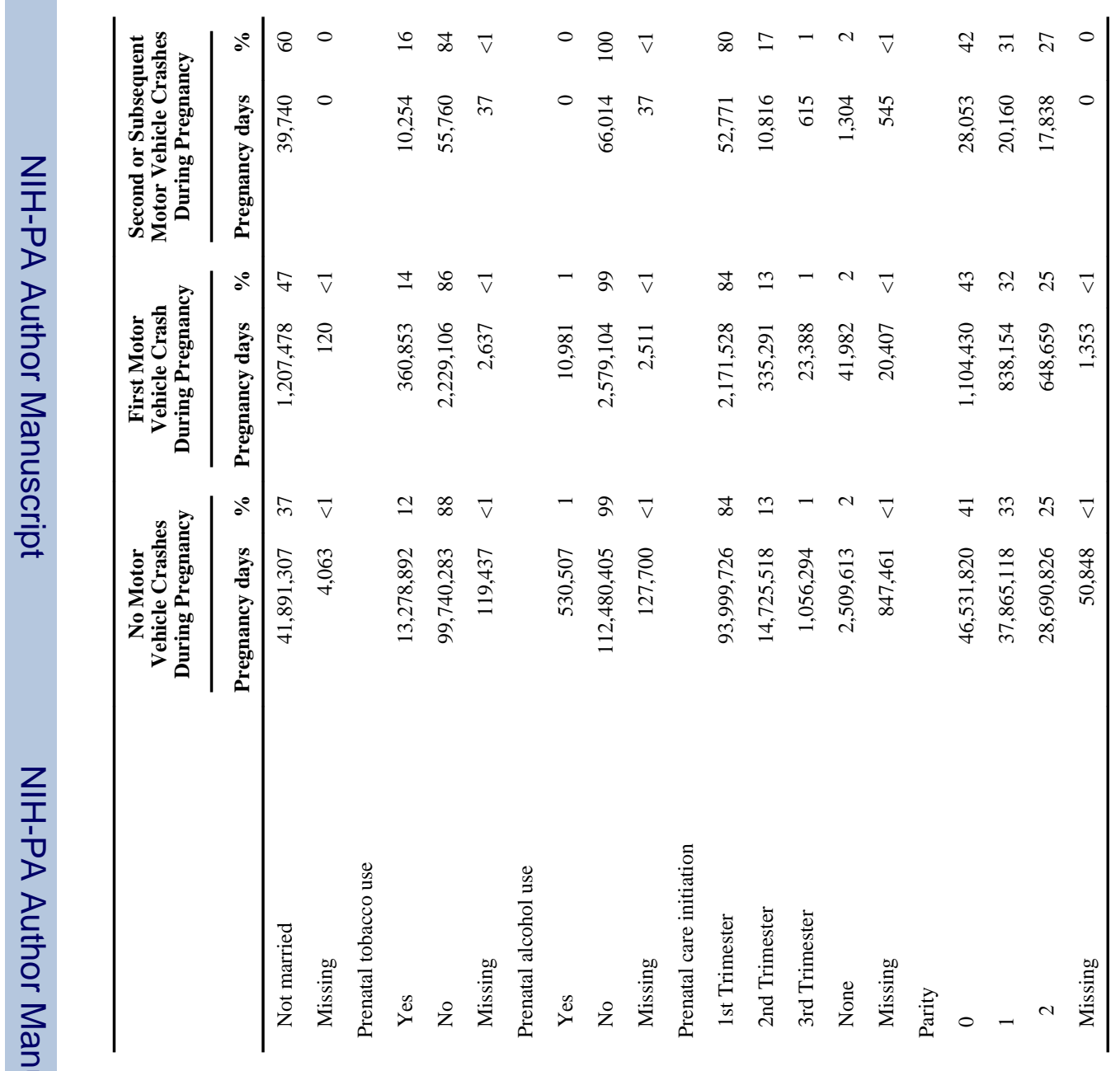




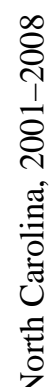

$\frac{\dot{s}}{\tilde{g}}$

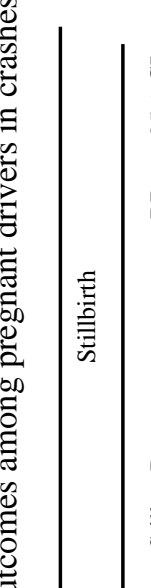

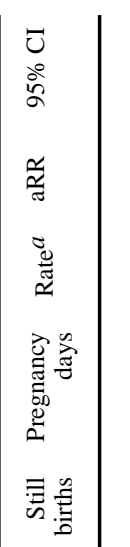

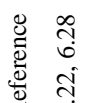

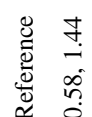

\& $\stackrel{8}{\text { i }}$

造

ํำ

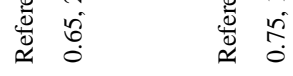

กั $\stackrel{9}{ \pm}$ क

$\stackrel{\infty}{-} \stackrel{8}{-} \stackrel{\circ}{\circ}$

तี่

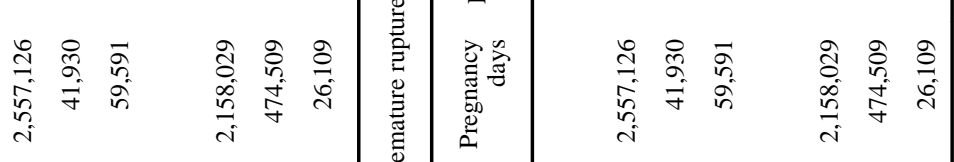

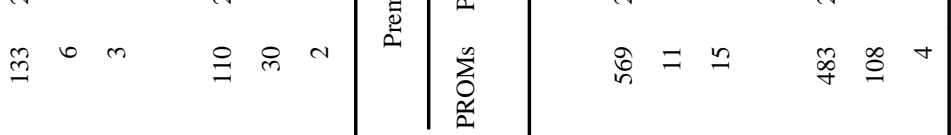

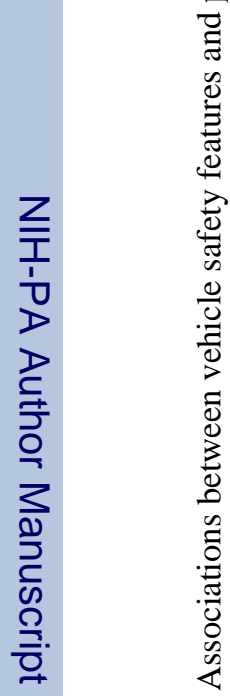
चี

可

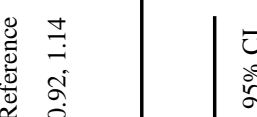

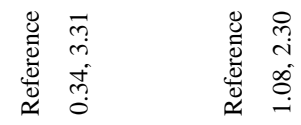

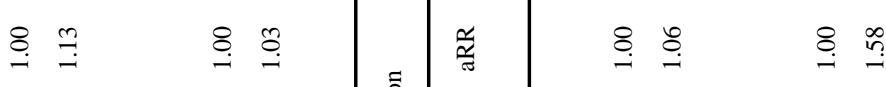

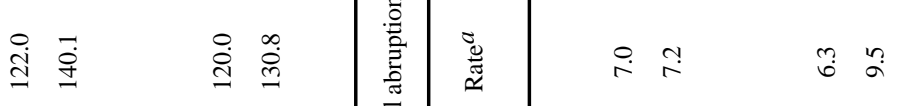

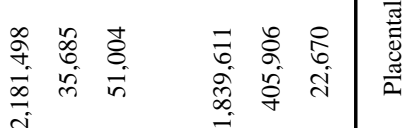

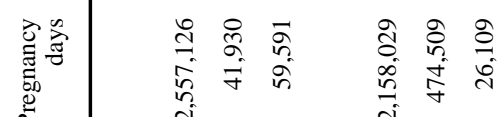

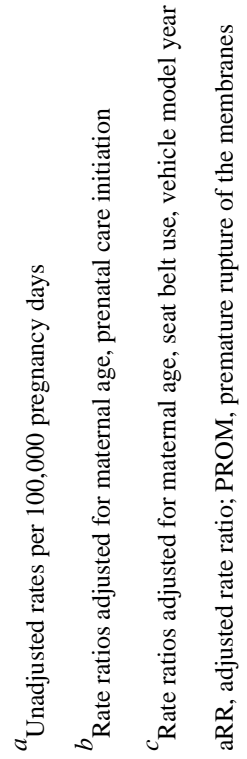
Am J Prev Med. Author manuscript; available in PMC 2014 November 01. 